\title{
UV protection and dermatological screening needed for IBD patients exposed to thiopurines
}

T he findings of a large, prospective, observational cohort study confirm that the exposure of patients with IBD to thiopurines increases the risk of nonmelanoma skin cancer (NMSC), regardless of whether the exposure is ongoing or was in the past. "The results support stringent UV protection and lifelong dermatological screening for thiopurine-treated IBD patients," says Laurent Peyrin-Biroulet, corresponding author for this sub-study of the CESAME (Cancers Et Surrisque Associé aux Maladies inflammatoires intestinales En France) study group in France.

The association between drug-induced immunosuppression and an increased risk of skin cancer among transplant recipients is well established. Until now, however, limited and conflicting data have been available on the risk of skin cancer in IBD patients exposed to immunosuppressants. Given the number of patients with IBD who are treated with azathioprine or mercaptopurine, Peyrin-Biroulet and colleagues decided to investigate whether azathioprine treatment was associated with an increased risk of NMSC in the CESAME IBD cohort, which was set up to capture the risk of any cancer or high-grade dysplasia.

19,486 consecutive IBD patients were enrolled in the CESAME cohort (by 608 French gastroenterologists) between May 2004 and June 2005. No exclusion criteria were applied. Data were collected electronically at interim visits and at the final visit, which was held during 2007. 16,459 patients were followed up at the final visit and the median follow-up time was 35 months. Only NMSC cases that developed after entry into the CESAME cohort were counted.

32 cases of NMSC were recorded: 20 basal-cell carcinomas and 12 squamouscell carcinomas. Of these 32 cases, 5 occurred in patients who had never received a thiopurine, 9 occurred in

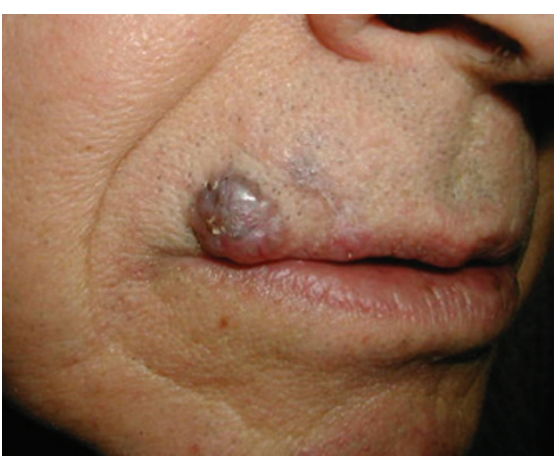

A pigmented basal-cell carcinoma $\odot$ Neville, J. A. et al. Nat. Clin. Pract. Oncol. 4, 462-469 (2007).

patients who had previously taken a thiopurine and 18 occurred in patients taking a thiopurine at the time NMSC was diagnosed. "Ongoing and past exposure to thiopurines significantly increases the risk of NMSC in IBD patients," explains Peyrin-Biroulet. Indeed, the authors reported that past or current use of thiopurines in the CESAME cohort conferred a sixfold to eightfold increase in the risk of developing NMSC, when compared with the risk for the general population (reference data were obtained from FRANCIM, the French Network of population-based Cancer Registries).

\section{4 ...exposure to thiopurines} significantly increases the risk of NMSC in IBD patients 77

In seven cases of NMSC that developed during the study period, other immunosuppressive drugs-infliximab, methotrexate and mycophenolate mofetil-were either being used at the time of NMSC diagnosis or had been used prior to the diagnosis. However, multivariate analysis adjusting for other immunosuppressive drugs did not affect the odds ratio for thiopurine exposure. Unsurprisingly, sun-exposed areas were the most frequent NMSC sites (15 patients developed NMSC on their face).
The study limitations noted by the authors include a lack of information on each patient's skin color, UV dose, smoking status, the dose and duration of thiopurine therapy, and the possible underestimation of incident cases of NMSC.

The authors also consider the mechanisms by which thiopurines may confer an increased risk of NMSC. They explain that DNA-damaging reactive oxygen species can be generated when thiopurines are exposed to UVA radiation. In addition, mutation of a candidate tumor suppressor gene $(P T C H)$ may result from azathioprine treatment.

"It is noteworthy," report the authors, "that the excess risk of NMSC found here was observable before the age of 50 , indicating that all IBD patients currently receiving, or having previously received, thiopurines should protect their skin from UV radiation and receive regular dermatological screening, regardless of their age." Further investigation is now needed to determine how efficacious UV protection and dermatological screening are at preventing the development of NMSC.

How patients with IBD who develop an NMSC while taking a thiopurine should be managed is unknown and, according to Peyrin-Biroulet, this poses new questions: "Should we switch to another drug?" and "Should we stop all immunosuppressive drugs?” In addition, as cancer multiplicity seems to be frequent in transplant recipients, whether or not this phenomenon holds true for IBD patients exposed to thiopurines needs to be researched.

Natalie J. Wood

Original article Peyrin-Biroulet, L. et al. Increased risk for non-melanoma skin cancers in patients who receive thiopurines for inflammatory bowel disease. Gastroenterology doi:10.1053/j.gastro.2011.06.050 